\title{
REAL HYPERSURFACES OF A COMPLEX MANIFOLD AND DISTRIBUTIONS WITH COMPLEX STRUCTURE
}

\author{
By Kentaro Yano ANd ShigerU Ishihara
}

The purpose of the present paper is to study real hypersurfaces of a complex manifold, distributions with complex structure induced on a real hypersurface from that of the ambient manifold, pseudo-conformal mappings between two real hypersurfaces and infinitesimal pseudo-conformol transformations on a real hypersurface.

In $\S 1$ we state some preliminaries on almost contact structures and an elementary lemma. In $\$ 2$ we study real hypersurfaces of an almost complex manifold and show that a hyperdistribution with complex structure is induced on the real hypersurface. We then show that, if we choose a local affine normal, there is on a real hypersurface an almost contact structure associated with the hyperdistribution endowed with complex structure. $\S 3$ is devoted to the study of affine connections induced on the real hypersurface form an affine connection of the ambient complex manifold with respect to which the complex structure is covariantly constant. In $\S 4$ we study pseudo-conformal mappings and in $\S 5$ infinitesimal pseudo-conformal transformations.

\section{$\S 1$. Preliminaries}

Let there be given, in a manifold $M$ of odd dimension $2 n+1(\geqq 3)$, a tensor field $f$ of type (1,1), a vector field $\xi$ and a 1 -form $\theta$ satisfying

$$
f^{2}=-I+\theta \otimes \xi, \quad f \xi=0, \quad \theta \circ f=0, \quad \theta(\xi)=1,
$$

$I$ being the identity tensor field of type $(1,1)$, or

$$
f_{e}^{a} f_{b}^{e}=-\delta_{b}^{a}+\theta_{b} \xi^{a}, \quad f_{e}^{a} \xi^{e}=0, \quad \theta_{e} f_{b}^{e}=0, \quad \theta_{e} \xi^{e}=1,
$$

$f_{b}{ }^{a}, \xi^{a}$ and $\theta_{b}$ denoting components of $f, \xi$ and $\theta$ respectively. Then the triple $(f, \xi, \theta)$ is called an almost contact structure in $M$. In the sequel, manifolds, tensor fields, connections and mappings we consider are assumed to be differentiable and of class $C^{\infty}$ unless otherwise stated and the indices $a, b, c, d, e, \cdots$ run over the range $\{1,2, \cdots, 2 n+1\}$, the summation convention being used with respect to this system of indices.

Received May 30, 1977 
We define tensor fields $S$ of type (1,2), $G$ of type $(0,2), T$ of type $(0,2), P$ of type $(1,1)$ and $Q$ of type $(0,1)$ as those with components

$$
\begin{aligned}
& S_{c b}^{a}=f_{c}^{e} \nabla_{e} f_{b}^{a}-f_{b}^{e} \nabla_{e} f_{c}^{a}-\left(\nabla_{c} f_{b}^{e}-\nabla_{b} f_{c}^{e}\right) f_{e}^{a}+\left(\nabla_{c} \theta_{b}-\nabla_{b} \theta_{c}\right) \xi^{a}, \\
& G_{c b}=f_{c}^{e}\left(\nabla_{e} \theta_{b}-\nabla_{b} \theta_{e}\right), \\
& T_{c b}=G_{c b}-G_{b c} \\
& P_{b}{ }^{a}=-\left[\xi^{e} \nabla_{e} f_{b}^{a}-\left(\nabla_{e} \xi^{a}\right) f_{b}^{e}+\left(\nabla_{b} \xi^{e}\right) f_{e}^{a}\right], \\
& Q_{b}=-\left[\xi^{e} \nabla_{e} \theta_{b}+\left(\nabla_{b} \xi^{e}\right) \theta_{e}\right],
\end{aligned}
$$

respectively, where $\nabla$ denotes the operator of covariant differentiation with respect to an arbitrary symmetric affine connection in $M$. We easily see that these tensor fields are independent of the symmetric connection $\nabla$ used to define them. Then $S$ and $G$ are respectively called the torsion tensor and the Levi tensor of $(f, \xi, \theta)$. The following propositions are well known [4]:

$$
\begin{aligned}
& S=0 \text { implies } T=0, \quad P=0 \text { and } Q=0 ; \\
& P=0 \text { implies } Q=0 .
\end{aligned}
$$

When the tensor field $S$ vanishes identically, the almost contact structure $(f, \xi, \theta)$ is said to be normal.

We now state an elementary lemma for later use. Let $V$ be a vector space over real number field with complex structure $F$. That is, $F: V \rightarrow V$ is a linear transformation satisfying $F^{2}=-I$. Then $V$ is necessarily even-dimensional, say $\operatorname{dim} V=2 n+2(\geqq 4)$. Take arbitrarily a $(2 n+1)$-dimensional subspace $W$ of $V$. Then $F W$ is also $(2 n+1)$-dimensional. We can now state

LEMMA 1.1. Put $D=W \cap F W$ and $N=F W-D$. Then $F D=D, F N \subset W, V$ $=W+F W, \operatorname{dim} D=2 n, N=\left\{a x_{0}+y \mid a \in R, a \neq 0, y \in D\right\}, x_{0}$ berng a fixed element of $N$, and any element $x$ of $N$ is uniquely represented as $x=a x_{0}+y(a \in R, y \in D)$.

The subset $N$ appearing in Lemma 1.1 has two connected components, each of which is homeomorphic to a Euclidean space of dimension $2 n+1$. The subset $N$ is called the affine normal space to $W$ in the vector space $V$ with complex structure $F$.

\section{$\S 2$. Hypersurfaces of an almost complex manifold.}

Let $\tilde{M}$ be an almost complex manifold of real dimension $2 n+2(\geqq 4)$ with almost complex structure $\tilde{F}$, where $\tilde{F}$ is a tensor field of type $(1,1)$ in $\tilde{M}$ satisfying $\widetilde{F}^{2}=-I$, i. e.

$$
\widetilde{F}_{k}^{h} \tilde{F}_{\imath}{ }^{k}=-\delta_{i}^{h},
$$


$\tilde{F}_{\imath}{ }^{h}$ denoting components of $\tilde{F}$. In the sequel, the indices $h, \imath, j, k, \cdots$ run over the range $\{1,2, \cdots, 2 n+2\}$ and the summation convention will be used with respect to this system of indices.

Let there be given a hypersurface $M$ immersed in $\tilde{M}$. For each point $P$ of $M$, denote the tangent space to $\tilde{M}$ and that to $M$ at $P$ by $T_{P}(\tilde{M})$ and $T_{P}(M)$ respectively. Then the subspace $D_{P}=T_{P}(M) \cap \tilde{F} T_{P}(M)$ is $2 n$-dimensional and hence the correspondence $P \mapsto D_{P}$ defines a distribution $D$ of dimension $2 n$ in $M$. Since $F D=D$, we can define a tensor field $J$ of type $(1,1)$ in $D$ by $J X=\widetilde{F} X, X$ being an arbitrary vector field belonging to $D$. Then $\widetilde{F}^{2}=-I$ implies $J^{2}=-I_{D}$, where $I_{D}$ denotes the identity tensor field of type $(1,1)$ in $D$. Thus the $D$ is called a hyperdistribution with complex structure $J$ in $M$ and said to be induced in $M$ from $\tilde{F}$ by the immersion [3].

Since the tangent space $T_{P}(\tilde{M})$ is a vector space with complex structure $\tilde{F}$, by Lemma 1.1 the subspace $T_{P}(M)$ of $T_{P}(\tilde{M})$ has its affine normal space $N_{P}$. We call $N=\bigcup_{P \in M} N_{P}$ the affine normal bundle to the hypersurface $M$. Since $N_{P}$ has two connected components, each of which is homeomorphic to a Euclidean space, $N$ has a global cross-section if $M$ is orientable.

Let $\tilde{U}$ be a coordinate neighborhood of $\tilde{M}$ such that any connected component $U$ of $\tilde{U} \cap M$ is a coordinate neighborhood of $M$. In the sequel by $U$ we mean such a coordinate neighborhood of $M$. Take a local cross-section $C$ of the affine normal bundle $N$ over $U$ and call it a local affine normal to $M$ in $U$. Then by Lemma $1.1 \tilde{F} C$ is tangent to $M$ in $U$ and hence

$$
\xi=-\tilde{F} C
$$

is a non-vanishing vector field in $U$. Next, for any vector field $X$ in $M$, we can decompose $\widetilde{F} X$ uniquely as

$$
\tilde{F} X=f X+\theta(X) C,
$$

where $f X$ is tangent to $M$. Thus $f$ and $\theta$ are a tensor field of type $(1,1)$ and a 1 -form in $U$ respectively. Applying $\tilde{F}$ to (2.3) and using $\tilde{F}^{2}=-I$, we find $-X$ $=\left(f^{2} X-\theta(X) \xi\right)+\theta(f X) C$, which implies

$$
f^{2}=-I+\theta \otimes \xi, \quad \theta \circ f=0 .
$$

If we put $X=\xi$ in (2.3), we obtain $\tilde{F} \xi=f(\xi)+\theta(\xi) C$. On the other hand (2.2) gives $\widetilde{F} \xi=C$. Hence we get

$$
f \xi=0, \quad \theta(\xi)=1 .
$$

Equations (2.4) and (2.5) show that the triple $(f, \xi, \theta)$ is an almost contact structure in $U$, which is called an almost contact structure induced $\imath n M$ by an affine normal $C$ in $U$. A vector field $X$ in $M$ belongs to $D$ if and only if $\widetilde{F} X$ belongs to $D$. Thus, because of (2.3), $X$ belongs to $D$ if and only if $\theta(X)=0$. Hence the distribution $D$ is defined by $\theta=0$ in $U$. Therefore the almost contact structure 
$(f, \xi, \theta)$ is associated with the hyperdis-tribution $D$ with complex structure [3].

We now take another affine normal $\bar{C}$ to $M$ in $U$. Then by Lemma 1.1 we have

$$
\bar{C}=\frac{1}{\alpha}(C+A),
$$

where $\alpha$ is a non-vanishing function and $A$ a vector field being tangent to $M$ and belonging to $D, \alpha$ and $A$ being defined in $U$. Thus we have

$$
\bar{f}=f-\theta \otimes A, \quad \bar{\xi}=\frac{1}{\alpha}(\xi-f A), \quad \bar{\theta}=\alpha \theta,
$$

where $(\bar{f}, \bar{\xi}, \bar{\theta})$ is the almost contact structure induced in $M$ by (2.3) and (2.5), $C$ being replaced by $\bar{C}$. The change (2.7) of almost contact structures has been discussed in [3] and is called a change of almost contact structures associated with $D$.

\section{$\S 3$. Induced affine connections}

We now assume that the ambient manifold $\tilde{M}$ is a complex manifold of complex dimension $n+1(\geqq 2)$ with complex structure $\tilde{F}$. It is well known that there is a symmetric affine connection $\tilde{\nabla}$ satisfying $\tilde{\nabla} \tilde{F}=0$, i. e.

$$
\tilde{\nabla}_{j} \tilde{F}_{\imath}{ }^{h}=0
$$

$[6],[7]$. In the sequel we fix this affine connection $\tilde{\nabla}$.

Consider a real hypersurface $M$ immersed in $\tilde{M}$ and a coordinate neighborhood $U$ of $M$ such that $U$ is a connected component of $\tilde{U} \cap M, \tilde{U}$ being a coordinate neighborhood of $\tilde{M}$. Let $\left(x^{h}\right)$ and $\left(y^{a}\right)$ be coordinates in $\tilde{U}$ and in $U$ respectively. We assume that $M$ is represented in $\tilde{U}$ by

$$
x^{h}=x^{h}\left(y^{a}\right) .
$$

Take an affine normal $C$ to $M$ in $U$ and put

$$
B_{b}{ }^{h}=\partial x^{h} / \partial y^{a}
$$

in $U$. Then $B_{b}=B_{b}{ }^{h} \partial / \partial x^{h}$ and $C=C^{h} \partial / \partial x^{h}$ form an affine $(2 n+2)$-frame along $U$. Thus on putting

$$
\left(\begin{array}{c}
B^{a}{ } \\
C_{\imath}
\end{array}\right)=\left(B_{b}{ }^{h}, C^{h}\right)^{-1},
$$

we have

$$
\begin{gathered}
B_{b}{ }^{\imath} B^{a}{ }_{i}=\delta_{b}^{a}, \quad B_{b}{ }^{\imath} C_{i}=0, \quad B^{a}{ }_{i} C^{\imath}=0, \quad C_{i} C^{\imath}=1 ; \\
B_{e}{ }^{h} B^{e}{ }+C^{h} C_{i}=\delta_{i}^{h} .
\end{gathered}
$$


Thus $B^{a}=B^{a}{ }_{i} d x^{2}$ and $C=C_{i} d x^{\imath}$ form a coframe dual to $\left\{B_{b}, C\right\}$ along $U$.

The affine connection $\nabla$ induced in $U$ from $\tilde{\nabla}$ with respect to the affine normal $C$ has, by definition, components given by

$$
\Gamma_{c b}^{a}=\left(\partial_{c} B_{b}{ }^{h}+\tilde{\Gamma}_{j i}^{h} B_{c}{ }^{j} B_{b}{ }^{i}\right) B^{a}{ }_{h},
$$

where $\partial_{b}=\partial / \partial y^{b}$ and $\tilde{\Gamma}_{j i}^{h}$ denote components of $\tilde{\nabla}$ in $\tilde{U}$. Since $\tilde{\nabla}$ is symmetric, i. e. since $\tilde{\Gamma}_{j i}^{h}=\tilde{\Gamma}_{\imath \jmath}^{h}, \nabla$ is also symmetric, i. e. $\Gamma_{c b}^{a}=\Gamma_{b c}^{a}$. Thus if we define the socalled van der Waerden- Bortolotti covariant derivative of $B_{b}{ }^{h}$ along $M$ by

$$
\nabla_{c} B_{b}{ }^{h}=\partial_{c} B_{b}{ }^{h}+\tilde{\Gamma}_{j i}^{h} B_{c}{ }^{j} B_{b}{ }^{2}-\Gamma_{c b}^{a} B_{a}{ }^{h}
$$

in $U$, then we have $\left(\nabla_{c} B_{b}{ }^{h}\right) B^{a}{ }_{h}=0$, which shows that $\nabla_{c} B_{b}{ }^{h}$ is of the form

$$
\nabla_{c} B_{b}{ }^{h}=h_{c b} C^{h},
$$

where $h_{c b}$ are defined by

$$
h_{c b}=h_{b c}=\left(\partial_{c} B_{b}{ }^{h}+\tilde{\Gamma}_{j i}^{h} B_{c}{ }^{j} B_{b}{ }^{i}\right) C_{h}
$$

and are called components of the covarnant second fundamental tensor $h$ of $M$ with respect to the affine normal $C, h$ being of type $(0,2)$.

Differentiating $B_{b}{ }^{h} B^{a}{ }_{h}=\delta_{b}^{a}$ covariantly along $M$ and using (3.8) and $C^{h} B^{a}{ }_{h}=0$, we find $B_{b}{ }^{h}\left(\nabla_{c} B^{a}{ }_{h}\right)=0$, from which

$$
\nabla_{c} B^{a}{ }_{i}=H_{c}{ }^{a} C_{\imath}
$$

where $\nabla_{c} B^{a}{ }_{\imath}$ are defined by

$$
\nabla_{c} B_{i}^{a}=\partial_{c} B^{a}{ }_{\imath}-\tilde{\Gamma}_{j i}^{h} B_{c}{ }^{j} B^{a}{ }_{h}+\Gamma_{c b}^{a} B^{b}{ }_{\imath}
$$

in $U$ and $H_{c}{ }^{a}$ by

$$
H_{c}{ }^{a}=-\left(\partial_{c} C^{h}+\tilde{\Gamma}_{j i}^{h} B_{c}{ }^{j} C^{i}\right) B^{a}{ }_{h} .
$$

The $H_{c}{ }^{a}$ are called components of the mixed second fundamental tensor $H$ of $M$ with respect to the affine normal $C$ in $U, H$ being of type (1.1).

We next differentiate $B^{a}{ }_{i} C^{\imath}=0$ covariantly along $M$ and use (3.10). Then we obtain $H_{c}{ }^{a}+B^{a}{ }_{i}\left(\nabla_{c} C^{i}\right)=0$, from which

$$
\nabla_{c} C^{h}=-H_{c}{ }^{a} B_{a}{ }^{h}+l_{c} C^{h},
$$

where $l_{c}$ are defined by

$$
l_{c}=\left(\partial_{c} C^{h}+\tilde{\Gamma}_{j i}^{h} B_{c}{ }^{j} C^{i}\right) C_{h}
$$

and $\nabla_{c} C^{h}$ by

$$
\nabla_{c} C^{h}=\partial_{c} C^{h}+\tilde{\Gamma}_{j i}^{h} B_{c}{ }^{j} C^{\imath}
$$

in $U$. The $l_{c}$ are called components of the thrrd fundamental tensor $l$ of $M$ with 
respect to the affine normal $C$ in $U, l$ being of type $(0,1)$. The $l$ gives a linear connection in the one-dimensional vector bundle

$$
\bigcup_{P \in U}\left\{a C_{P} \mid a \in R\right\} \quad \text { over } U \text {. }
$$

Finally, differentiating $B_{b}{ }^{2} C_{i}=0$ covariantly along $M$ and using (3.8), $C_{i} B_{b}{ }^{2}=0$ and $C_{i} C^{\imath}=1$, we find $l_{c}+C^{\imath}\left(\nabla_{c} C_{\imath}\right)=0$, from which

$$
\nabla_{c} C_{i}=-h_{c b} B^{b}{ }_{\imath}-l_{c} C_{\imath},
$$

where $\nabla_{c} C_{\imath}$ are defined in $U$ by

$$
\nabla_{c} C_{i}=\partial_{c} C_{\imath}-\tilde{\Gamma}_{j i}^{h} B_{c}{ }^{j} C_{h} .
$$

Equations (3.8) and (3.10) are those of Gauss for the real hypersurface $M$ and equations (3.11) and (3.13) are those of Weingarten for $M$.

Consider a vector field $X=X^{h} \partial / \partial x^{h}$ tangent to $M$. Then we have $X^{h}=X^{a} B_{a}{ }^{h}$. Thus using (3.8), we have

$$
\nabla_{c} X^{h}=\left(\nabla_{c} X^{a}\right) B_{a}{ }^{h}+h_{c b} X^{b} C^{h},
$$

where we have put in $U$

$$
\nabla_{c} X^{h}=\partial_{c} X^{h}+\tilde{\Gamma}_{j i}^{h} B_{c}{ }^{\jmath} X^{\imath}, \quad \nabla_{c} X^{a}=\partial_{c} X^{a}+\Gamma_{c b}^{a} X^{b} .
$$

Let $(f, \xi, \theta)$ be the almost contact structure induced in $M$ by the affine normal $C$ to $M$ in $U$. Then (2.2) and (2.3) can be written as

$$
\begin{aligned}
& \widetilde{F}_{\imath}{ }^{h} C^{\imath}=-\xi^{b} B_{b}{ }^{h}, \\
& \tilde{F}_{\imath}{ }^{h} B_{b}{ }^{2}=f_{b}{ }^{a} B_{a}{ }^{h}+l_{b} C^{h}
\end{aligned}
$$

respectively. Applying $\nabla_{c}$ to (3.20) and using $\nabla_{c} \widetilde{F}_{\imath}{ }^{h}=B_{c}{ }^{j} \tilde{\nabla}_{j} \widetilde{F}_{\imath}{ }^{h}=0$, we obtain

$$
h_{c b}\left(-f^{a} B_{a}{ }^{h}\right)=\left(\nabla_{c} f_{b}{ }^{a}\right) B_{a}{ }^{h}+h_{c e} f_{b}{ }^{e} C^{h}+\left(\nabla_{c} \theta_{b}\right) C^{h}+\theta_{b}\left(-H_{c}{ }^{a} B_{a}{ }^{h}+l_{c} C^{h}\right),
$$

where we have used (2.2), (2.3) with $X=B_{b}$, (3.16) and (3.17). Thus we obtain

$$
\begin{aligned}
& \nabla_{c} f_{b}{ }^{a}=-h_{c b} \xi^{a}+H_{c}{ }^{a} \theta_{b}, \\
& \nabla_{c} \theta_{b}=-h_{c e} f_{b}{ }^{e}-l_{c} \theta_{b} .
\end{aligned}
$$

Next, applying $\nabla_{c}$ to (3.19), we have in a similar way as above

$$
-H_{c}{ }^{e}\left(f_{e}{ }^{a} B_{a}{ }^{h}+\theta_{e} C^{h}\right)+l_{c}\left(-\xi^{a} B_{a}{ }^{h}\right)=-\left(\nabla_{c} \xi^{a}\right) B_{a}{ }^{h}-h_{c e} \xi^{e} C^{h},
$$

from which

$$
\begin{gathered}
\nabla_{c} \xi^{a}=f_{a}^{a} H_{c}{ }^{e}+l_{c} \xi^{a}, \\
H_{c}{ }^{e} \theta_{e}=h_{c e} \xi^{e} .
\end{gathered}
$$


Substituting (3.21), (3.22) and (3.23) into (1.3) and using (3.24), we obtain

$$
\begin{gathered}
S_{c b}{ }^{a}=\left(H_{e}{ }^{a} f_{c}^{e}-f_{e}{ }^{a} H_{c}{ }^{e}-l_{c} \xi^{a}\right) \theta_{b}-\left(H_{e}{ }^{a} f_{b}{ }^{e}-f_{e}{ }^{a} H_{b}{ }^{e}-l_{b} \xi^{a}\right) \theta_{c}, \\
G_{c b}=-\left(h_{c b}+f_{c}{ }^{e} f_{b}{ }^{d} h_{e d}\right)+h_{b e} \xi^{e} \theta_{c}-f_{c}{ }^{e} l_{e} \theta_{b}, \\
P_{b}{ }^{a}=-\left(H_{b}{ }^{a}+f_{b}{ }^{e} f_{d}{ }^{a} H_{e}{ }^{d}\right)+H_{e}{ }^{a} \xi^{e} \theta_{b}-f_{b}{ }^{e} l_{c} \xi^{a}, \\
Q_{b}=l_{b}-\left(l_{e} \xi^{e}\right) \theta_{b}-h_{e d} \xi^{e} f_{b}{ }^{d} .
\end{gathered}
$$

When a hyperdistribution $D$ with complex structure $J$ is given in a manifold of odd dimension and when $S_{c b}{ }^{a} \equiv 0,\left(\bmod \theta_{c}, \theta_{b}\right)$ is satisfied for an almost contact structure $(f, \xi, \theta)$ associated with $D$, the $D$ is said to be torsionless [3]. Thus we have from (3.25)

THEOREM 3.1. For any real hypersurface $M$ of a complex manifold the induced hyperdistribution $D$ of $M$ with complex structure $J$ is always torsionless.

Equations (3.26) imply

THEOREM 3.2. For any real hypersurface $M$ of a complex manifold, the Levi tensor $G$ of an almost contact structure $(f, \xi, \theta)$ induced $2 n M$ has components of the form

$$
G_{c b}=-\left(h_{c b}+f_{c}{ }^{e} f_{b}{ }^{d} h_{e d}\right) \quad\left(\bmod . \theta_{c}, \theta_{b}\right)
$$

in $U$, when an affine normal $C$ to $M$ is given in a coordinate neighborhood $U$ of $M$.

Theorem 3.2 implies that

$$
G(X, Y)=G(Y, X), \quad G(J X, J Y)=G(X, Y)
$$

for any vector fields $X$ and $Y$ belonging to the hyperdistribution $D$ with complex structure $J$. Equations (3.25) imply

THEOREM 3.3. Let $(f, \xi, \theta)$ be an almost contact structure induced on a real hypersurface $M$ of a complex manifold by giving an affine normal $C$ to $M$ in a coordinate neighborhood $U$ of $M$. Then $(f, \xi, \theta)$ is normal if and only if

$$
H_{e}{ }^{a} f_{b}{ }^{e}-f_{e}{ }^{a} H_{b}{ }^{e}-l_{b} \xi^{a} \equiv 0, \quad\left(\bmod \theta_{b}\right) .
$$

We take another affine normal $\bar{C}$ to $M$ in $U$ and assume $\bar{C}$ is given by (2.6). Denote by $\bar{\nabla}, \bar{l}, \bar{h}$ and $\bar{H}$ respectively the induced affine connection, the third fundamental tensor, the covariant and the mixed second fundamental tensors of $M$ in $U$, which are determined by (3.6), (3.14), (3.9) and (3.12) in terms of $\bar{C}$. Then components $\bar{\Gamma}_{c b}^{a}$ of $\bar{\nabla}, \bar{h}_{c b}$ of $\bar{h}, \bar{H}_{b}{ }^{a}$ of $\bar{H}$ and $\bar{l}_{b}$ of $\bar{l}$ are respectively given by

$$
\begin{aligned}
& \bar{\Gamma}_{c b}^{a}=\Gamma_{c b}^{a}-h_{c b} A^{a}, \quad \bar{h}_{c b}=\alpha h_{c b}, \\
& \bar{H}_{b}{ }^{a}=\frac{1}{\alpha}\left[H_{b}{ }^{a}-\nabla_{b} A^{a}+\left(l_{b}+h_{b e} A^{e}\right) A^{a}\right],
\end{aligned}
$$




$$
\bar{l}_{b}=l_{b}+h_{b e} A^{e}-\nabla_{b} \log |\alpha| \text {, }
$$

where $\alpha$ is a non-vanishing function and $A=A^{a} B_{a}{ }^{h} \partial / \partial x^{h}$ is a vector field belonging to $D$, both being defined in $U$. To obtain (3.31), we have used (2.6), $\bar{B}_{b}{ }^{h}=$ $B_{b}{ }^{h}$ and

$$
\bar{B}_{i}^{a}=B^{a}{ }_{\imath}-A^{a} C_{\imath}, \quad \bar{C}_{i}=C_{\imath},
$$

where

$$
\left(\begin{array}{c}
\bar{B}^{a}{ }_{\imath} \\
\bar{C}_{\imath}
\end{array}\right)=\left(\bar{B}_{a}{ }^{h}, \bar{C}^{h}\right)^{-1} .
$$

Theorem 3.2 and $\bar{h}_{c b}=\alpha h_{c b}$ appearing in (3.21) imply the following well known theorem [1], [2], [3], [5]:

THEOREM 3.4. Let $(f, \xi, \theta)$ and $(\bar{f}, \bar{\xi}, \bar{\theta})$ be two almost contact structures induced on a real hypersurface $M$ and assume that they are related to each other by (2.7). Then

$$
\bar{G}_{c b} \equiv \alpha G_{c b} \quad\left(\bmod \theta_{c}, \theta_{b}\right),
$$

$\alpha$ being a non-vanishng function, where $G_{c b}$ and $\bar{G}_{c b}$ are respectively components of the Levi tensors of $(f, \xi, \theta)$ and $(\bar{f}, \bar{\xi}, \bar{\theta})$.

Theorem 3.4 shows that the restriction $G_{D}$ of the Levi tensor $G$ to $D$ is determined up to a non-vanishing factor. Thus $G_{D}$ is sometimes called the Levi tensor of the induced hyperdistribution $D$ with complex structure. When $G$ is of the maximum rank $2 n$ everywhere in $M$, the real hypersurface $M$ is said to be non-degenerate. By Theorem 3.1, for any real hypersurface $M$ of a complex manifold the hyperdistribution $D$ of $M$ with complex structure is torsionless. This fact means that any real hypersurface $M$ admits a pseudo-conformal structure when $M$ is non-degenerate [1], [2], [5].

\section{$\S 4$. Pseudo-conformal mappings.}

Let $M$ and ' $M$ be two manifolds admitting hyperdistributions $D$ and ' $D$ with complex structures $J$ and ' $J$ respectively. Assume that there is a homeomorphism $\phi: M \rightarrow^{\prime} M$ such that, for any vector field $X$ belonging to $D, \phi_{*} X$ belongs to ' $D$ and $\phi_{*} J=^{\prime} J \phi_{*}$, where $\phi_{*}$ denotes the differential of $\phi$. Then $\phi: M \rightarrow^{\prime} M$ is called a pseudo-conformal mapping [3], [5].

Let $\tilde{\phi}: \tilde{M} \rightarrow \tilde{M}$ be a holomorphic transformation of the ambient complex manifold $\tilde{M}$ with complex structure $\tilde{F}$. Then $\tilde{\phi}_{*} \tilde{F}=\tilde{F} \tilde{\phi}_{*}$, where $\tilde{\phi}_{*}$ denotes the differential of $\tilde{\phi}$. Consider real hypersurfaces $M$ and ' $M$ immersed in $\tilde{M}$ and assume $\tilde{\phi}(M)=^{\prime} M$. Denote by $\phi: M \rightarrow^{\prime} M$ the restriction of $\tilde{\phi}$ to $M$. Then $\phi$ is a homeomorphism and is called the mapping induced from $\tilde{\phi}$. Let $D$ and ' $D$ be the hyperdistributions with complex structure induced in $M$ and ' $M$ respec- 
tively. Denote by $J$ and ' $J$ the complex structures induced in $D$ and ' $D$ respectively. Then we can easily verify that $\phi_{*} X$ belongs to ' $D$ whenever $X$ belongs to $D$ and that $\phi_{*} J=^{\prime} J \phi_{*}$. Thus $\phi: M \rightarrow^{\prime} M$ is a pseudo-conformal mapping. Hence we have the following well known theorem [1], [2], [5]:

THEOREM 4.1. Any holomorphic transformation $\tilde{\phi}: \tilde{M} \rightarrow \tilde{M}$ of the ambient complex manifold $\tilde{M}$ induces a pseudo-conformal mapping $\phi: M \rightarrow ' M$, where $M$ and ' $M$ are real hypersurfaces in $\tilde{M}$ such that ' $M=\tilde{\phi}(M)$.

Let $\tilde{\phi}: \tilde{M} \rightarrow \tilde{M}$ and $\phi: M \rightarrow^{\prime} M$ be taken as above. If we take an affine normal $C$ to $M$ in a coordinate neighborhood $U$ of $M$, then $\bar{C}=\tilde{\phi}_{*}(C)$ is also an affine normal to ' $M$ in ' $U=\phi(U)$ because of $\tilde{\phi}_{*} \tilde{F}=\tilde{F} \tilde{\phi}_{*}$. Thus, taking an affine normal ' $C$ to ' $M$ in ' $U$, we get because of (2.6)

$$
\bar{C}=\frac{1}{\alpha}\left({ }^{\prime} C+A\right)
$$

in ' $U$, where $\alpha$ is a non-vanishing function and $A$ a vector field belonging to ' $D$, both being defined in ' $U$. Let $(f, \xi, \theta)$ be the almost contact structure induced in $M$ by the affine normal $C$ to $M$ in $U$. Let $\left({ }^{\prime} f,{ }^{\prime} \xi,^{\prime}, \theta\right)$ be the almost contact structure induced in ' $M$ by the affine normal ${ }^{\prime} C$ to ' $M$ in ' $U$. Then putting

$$
\bar{f}=\phi_{*} f\left(\phi_{*}\right)^{-1}, \quad \bar{\xi}=\phi \xi, \quad \bar{\theta}=\theta \circ \phi,
$$

we see that $(\bar{f}, \bar{\xi}, \bar{\theta})$ is an almost contact structure associated with ' $D$ in ' $U$. Thus, taking account of (2.7), we have from (4.1)

$$
\bar{f}==^{\prime} f-^{\prime} \theta \otimes A, \quad \bar{\xi}=\frac{1}{\alpha}\left(\xi-{ }^{\prime} f A\right), \quad \bar{\theta}=\alpha^{\prime} \theta .
$$

In general, the following lemma prevails [3]:

LEMMA 4.2. For a homeomorphism $\phi \quad M \rightarrow$ 'M of a manifold $M$ admitting a hyperdistribution with complex structure onto another ' $M$, (4.3) is a necessary and sufficient condition for $\phi^{\cdot} M \rightarrow^{\prime} M$ to be a pseudo-conformal mapping.

Let $\tilde{\phi}: \tilde{M} \rightarrow \tilde{M}, \phi: M \rightarrow^{\prime} M$ and other notions be fixed as above. Take a coordinate neighborhood $\tilde{U}$ of $\tilde{M}$ with coordinates $\left(x^{h}\right)$ and put ' $\tilde{U}=\tilde{\phi}(\tilde{U})$, which is a coordinate neighborhood of $\tilde{M}$ with coordinates $\left(u^{h}\right)$. Then $\tilde{\phi}: \tilde{M} \rightarrow \tilde{M}$ will be locally represented by

$$
u^{h}=\widetilde{\Phi}^{h}\left(x^{i}\right)
$$

with respect to $\tilde{U}$ and ' $\tilde{U}$. If we take complex coordinates $\left(z^{\lambda}\right)$ and $\left(w^{\lambda}\right)$ in $\tilde{U}$ and ' $\tilde{U}$ respectively, then (4.4) is represented as follows:

$$
w^{\lambda}=\tilde{\Phi}^{\lambda}\left(z^{\mu}, z^{\bar{\mu}}\right),
$$

where $z^{\bar{\mu}}=\overline{z^{\mu}}$. In the sequel the indices $\lambda, \mu, \nu, \cdots$ run over the range $\{1,2, \cdots$, $n+1$. Then we can easily prove 
LEMMA 4.3. The condition $\tilde{\phi}_{*} \tilde{F}=\tilde{F} \tilde{\phi}_{*}$ is equivalent to each of the following conditions (4.6), (4.7) and (4.8):

$$
\left[\frac{\partial \check{\Phi}^{h}}{\partial x^{k}} \tilde{F}_{\imath}{ }^{k}\right]_{P}=\left[{ }^{\prime} F_{k}{ }^{h} \frac{\partial \tilde{\Phi}^{k}}{\partial x^{\imath}}\right]_{\phi(P)}
$$

for any point $P$ of $\tilde{U}$ where $\tilde{F}_{\imath}{ }^{h}$ and ${ }^{\prime} \tilde{F}_{2}{ }^{h}$ are components of $\tilde{F}$ in $\tilde{U}$ and in ' $\tilde{U}$ respectively:

$$
\begin{gathered}
\frac{\partial \tilde{\Phi}^{\lambda}}{\partial \bar{z}^{\mu}}=0 ; \\
d \tilde{\Phi}^{\lambda} \wedge d z^{1} \wedge d z^{2} \wedge \cdots \wedge d z^{n+1}=0 .
\end{gathered}
$$

Denote by $\left(y^{a}\right)$ and $\left(\eta^{a}\right)$ coordinates of $M$ in $U$ and those of ' $M$ in ' $U$ respectively. Then $\phi^{-1}:{ }^{\prime} M \rightarrow M$ will be represented by

$$
y^{a}=\psi^{a}\left(\eta^{b}\right)
$$

with respect to $U$ and ' $U$. Moreover the hypersurfaces $M$ and ' $M$ are assumed to be represented by

$$
x^{h}=x^{h}\left(y^{a}\right) \text { and } u^{h}=u^{h}\left(\eta^{a}\right)
$$

respectively with respect to the pairs $(U, \tilde{U})$ and $(' U, ' \tilde{U})$. Putting

we obtain

$$
B_{b}{ }^{h}=\frac{\partial x^{h}}{\partial u^{b}} \text { in } U \text { and } \quad B_{b}{ }^{h}=\frac{\partial u^{h}}{\partial \eta^{b}} \quad \text { in } ' U,
$$

LEMMA 4.4. The condition (4.3) for the $\phi \quad M \rightarrow{ }^{\prime} M$ ' which is induced from $\tilde{\phi}: \tilde{M} \rightarrow \tilde{M}$ is equivalent to each of the following conditions (4.11) and (4.12):

$$
\left[\frac{\partial \tilde{\Phi}^{h}}{\partial x^{k}} \widetilde{F}_{\imath}{ }^{k} B_{e}{ }^{2} \frac{\partial \psi^{e}}{\partial \eta^{b}}\right]_{P}=\left[{ }^{\prime} F_{\imath}{ }^{\prime \prime} B_{b}{ }^{2}\right]_{\phi(P)}
$$

for any point $P$ of $U$;

$$
d\left(\tilde{\Phi}^{\lambda} \circ i\right) \wedge d\left(z^{1} \circ i\right) \wedge \cdots \wedge d\left(z^{n+1} \circ i\right)=0,
$$

where $\imath: M \rightarrow \tilde{M}$ denotes the immersion of $M$.

Proof. Denote components of $C$ in $\tilde{U}$, those of ' $C$ in ' $\tilde{U}$ and those of $\bar{C}$ in ${ }^{\prime} \tilde{U}$ by $C^{h},{ }^{\prime} C^{h}$ and $\bar{C}^{h}$ respectively. Then we have for any point $P$ of $U$

$$
\left[\frac{\partial \tilde{\Phi}^{h}}{\partial x^{k}} C^{k}\right]_{P}=\left[C^{h}\right]_{\varphi(P)}
$$

On the other hand we easily obtain

$$
\left[\frac{\partial \widetilde{\Phi}^{h}}{\partial x^{k}} B_{e}{ }^{k} \frac{\partial \psi^{e}}{\partial \eta^{b}}\right]_{P}=\left[{ }^{\prime} B_{b}{ }^{h}\right]_{\phi(P)} .
$$


Transvecting (4.6) with $\left[B_{e}^{\imath} \frac{\partial \psi^{e}}{\partial \eta^{b}}\right]_{P}$ and using (4.13) and (4.14), we obtain

$$
\left[\frac{\partial \tilde{\Phi}^{h}}{\partial x^{k}} \widetilde{F}_{\imath}{ }^{k} B_{e}{ }^{2} \frac{\partial \varphi^{e}}{\partial \eta^{b}}\right]_{P}=\left[{ }^{\prime} \widetilde{F}_{\imath}{ }^{\prime \prime} B_{b}{ }^{2}\right]_{\phi(P)},
$$

which is equivalent to

$$
\left[\left(\frac{\partial \psi^{e}}{\partial \eta^{b}} f_{e}^{a}\right)^{\prime} B_{a}{ }^{h}+\left(\frac{\partial \psi^{e}}{\partial \eta^{b}} \theta_{e}\right) \bar{C}^{h}\right]_{\phi(P)}=\left[\left({ }^{\prime} f_{b}{ }^{e} \frac{\partial \psi^{a}}{\partial \eta^{e}}\right)^{\prime} B_{a}{ }^{h}+{ }^{\prime} \theta_{b}{ }^{\prime} C^{h}\right]_{\phi(P)} .
$$

Equation (4.16) is equivalent to (4.3). Thus (4.15) is equivalent to (4.3). Finally, if we take account of Lemma 4.3 and (4.14), we see easily that (4.15) is equivalent to (4.12). Therefore Lemma 4.4 is proved.

Let there be given abstractly a homeomorphism $\phi: M \rightarrow^{\prime} M$ and assume that $\phi$ is represented by

$$
u^{h}=\hat{\Phi}^{h}\left(y^{a}\right) \quad \text { or } \quad w^{\lambda}=\hat{\Phi}^{\lambda}\left(y^{a}\right)
$$

with respect to $U$ and ' $U$. Then in a way similar to that used for the proof of Lemma 4.4 we can prove

LEMMA 4.5. For any homeomorphism $\phi: M \rightarrow^{\prime} M$, which is abstractly given, the condition (4.3) is equivalent to the condition

$$
d \Phi^{\lambda} \wedge d\left(z^{1} \circ i\right) \wedge \cdots \wedge d\left(z^{n+1} \circ i\right)=0,
$$

where $i \cdot M \rightarrow \tilde{M}$ is the immersion of $M$.

We now assume that $M$ and ' $M$ are real hypersurfaces analytically immersed in $\tilde{M}$ and that $\phi: M \rightarrow^{\prime} M$ is an analytic homeomorphism. Then, as is well known, the differential equation (4.8) with unknown functions $\widetilde{\Phi}^{\lambda}\left(z^{\mu}, z^{\bar{\mu}}\right)$ has a local solution $\widetilde{\Phi}^{\lambda}$ satisfying the boundary condition

$$
\tilde{\Phi}^{\lambda} \circ i=\hat{\Phi}^{\lambda}
$$

along $M$, when $\Phi^{\lambda}$ satisfy the condition (4.18) [5]. Therefore, taking account of Theorem 4.1, Lemmas 4.2, 4.3, 4.4 and 4.5, we can prove the following well known theorem [1], [2], [5]:

THEOREM 4.6. Let $M$ and ' $M$ be real hypersurfaces analytically immersed $i n$ a complex manifold $\tilde{M}$ and assume that $\phi \cdot M \rightarrow^{\prime} M$ is an analytic homeomorphism. Then $\phi$ is pseudo-conformal if and only if, for any point $P$ belonging to $M$, there are neighborhoods $\tilde{O}$ and ' $\tilde{O}$ of $\tilde{M}$ containing respectively $P$ and $\phi(P)$ and a holomorphic homeomorphism $\tilde{\phi}: \tilde{O} \rightarrow ' \tilde{O}$ such that $\phi$ is the restruction of $\tilde{\phi}$ to $\tilde{O} \cap M$.

\section{§. Infinitesimal pseudo-conformal transformations.}

Let $X$ be a vector field in a manifold $M$ admitting a hyperdistribution $D$ 
with complex structure and assume that any local transformations $\phi_{t}(-\varepsilon<t<\varepsilon$, $\varepsilon>0$ ) of $M$ generated by $X$ are always pseudo-conformal transformations. Then $X$ is called an infinitesimal pseudo-conformal transformation or simply a pseudoconformal vector field in $M$. Let $(f, \xi, \theta)$ be an almost contact structure associated with $D$ in a coordinate neighborhood $U$. Then we have the following lemma proved in [3] for a manifold admitting a hyperdistribution with complex structure :

LEMMA 5.1. In a real hypersurface $M$ of a complex manifold, a vector field $X$ is pseudo-conformal if and only if $X$ satısfies

$$
\mathcal{L}_{X} f=-\theta \otimes V, \quad \mathcal{L}_{X} \xi=-a \xi-f V, \quad \mathcal{L}_{X} \theta=a \theta,
$$

where $a$ is a function and $V$ a vector field belonging to $D$, both being defined in $U$.

It is known that a pseudo-conformal vector field $X$ in $M$ vanishes identically if $X$ belongs to $D$, where $D$ is assumed to be torsionless and non-degenerate. On the other hand, by Theorem 3.1, for any real hypersurface $M$ of a complex manifold $\tilde{M}$ the induced hyperdistribution $D$ of $M$ is always torsionless. Thus we have [5]

THEOREM 5.2. Let $M$ be a non-degenerate real hypesurface of a complex manıfold $\tilde{M}$. A pseudo-conformal vector field $X$ in $M$ vanishes identically if $X$ belongs to the induced hyperdistribution $D$ with complex structure.

Consider a real hypersurface $M$ of a complex manifold $\tilde{M}$ with complex structure $\tilde{F}$. Let a holomorphic vector field $\tilde{X}$ in $\tilde{M}$ be tangent to $M$. Then, since $X$ is holomorphic, $X$ satisfies

$$
\tilde{F}_{k}{ }^{h} \tilde{\nabla}_{\imath} \tilde{X}^{k}=\tilde{V}_{k} \tilde{X}^{h} \tilde{F}_{\imath}{ }^{k}
$$

On the other hand, since $\tilde{X}$ is tangent to $M$, we have along $M$

$$
\tilde{X}^{h}=X^{a} B_{a}{ }^{h} \text {. }
$$

Transvection of (5.2) with $B_{b}{ }^{2}$ gives

$$
\tilde{F}_{k}{ }^{h}\left(\tilde{\nabla}_{\imath} \tilde{X}^{k}\right) B_{b}{ }^{2}=\left(\tilde{\nabla}_{k} \tilde{X}^{h}\right) \tilde{F}_{\imath}{ }^{k} B_{b}{ }^{\imath},
$$

which is equivalent to

$$
\begin{aligned}
{\left[\left(\nabla_{b} X^{e}\right) f_{e}{ }^{a}\right.} & \left.-\left(\nabla_{e} X^{a}\right) f_{b}{ }^{e}-h_{b e} X^{e} \xi^{a}\right] B_{a}{ }^{h} \\
& +\left[\left(\nabla_{b} X^{e}\right) \theta_{e}-f_{b}{ }^{e} h_{e d} X^{d}\right] C^{h}=\theta_{b}\left(C^{k} \tilde{\nabla}_{k} \tilde{X}^{h}\right)
\end{aligned}
$$

because of (3.18), (3.19), (3.20) and (5.3), where $(f, \xi, \theta)$ is an almost contact structure induced in each coordinate neighborhood $U$ of $M$ by fixing an affine normal $C$ to $M$ in $U$. Next, transvection of (5.2) with $C^{2}$ gives

$$
C^{i} \tilde{\nabla}_{\imath} \tilde{X}^{h}=\xi^{c}\left[\left(\nabla_{c} X^{e}\right) f_{e}{ }^{a}-h_{c e} X^{e} \xi^{a}\right] B_{a}{ }^{h}+\left[\xi^{c}\left(\nabla_{c} X^{e}\right) \theta_{e}\right] C^{h}
$$


because of (3.18), (3.19), (3.20) and (5.3). Substituting (5.6) into (5.5), we have

$$
\begin{aligned}
& \left(\nabla_{b} X^{e}\right) f_{e}^{a}-\left(\nabla_{e} X^{a}\right) f_{b}^{e}-h_{b e} X^{e} \xi^{a}=\theta_{b}\left[\xi^{c}\left(\nabla_{c} X^{e}\right) f_{e}{ }^{a}-h_{c e} \xi^{c} X^{e} \xi^{a}\right], \\
& \left(\nabla_{b} X^{e}\right) \theta_{e}-f_{b}{ }^{e} h_{e d} X^{d}=\theta_{b}\left(\nabla_{c} X^{e}\right) \xi^{c} \theta_{e},
\end{aligned}
$$

which reduce respectively to

$$
\mathcal{L}_{X} f_{b}^{a}=-\theta_{b} V^{a}, \quad \mathcal{L}_{X} \theta_{b}=a \theta_{b},
$$

where we have put

$$
\begin{aligned}
& V^{a}=-H_{e}^{a} X^{e}+h_{c e} \xi^{c} X^{e} \xi^{a}-\xi^{c}\left(\nabla_{c} X^{e}\right) f_{e}^{a}, \\
& a=\left(\nabla_{c} X^{e}\right) \xi^{c} \theta_{e}-l_{e} X^{e} .
\end{aligned}
$$

Thus, taking account of (3.24), we see easily that $\theta_{e} V^{e}=0$, i. e. that $V^{a}$ are components of a vector field $V$ belonging to the induced hyperdistribution $D$ of $M$. Next, the identities $\theta_{b} \xi^{b}=1$ and $f_{b}{ }^{a} \xi^{b}=0$ imply respectively

$$
\left(\mathcal{L}_{X} \theta_{b}\right) \xi^{b}+\theta_{b}\left(\mathcal{L}_{X} \xi^{b}\right)=0 \text { and }\left(\mathcal{L}_{X} f_{b}^{a}\right) \xi^{b}+f_{b}^{a}\left(\mathcal{L}_{X} \xi^{b}\right)=0
$$

Substituting (5.8) into these equations, we obtain

$$
\mathcal{L}_{X} \xi^{a}=-a \xi^{a}-f_{e}^{a} V^{e} .
$$

Consequently, we have (5.1) from (5.8) and (5.10). Thus we have the following theorem [5]:

THEOREM 5.3. Let $M$ be a real hypersurface immersed in a complex manrfold. If a holomorphic vector field $\tilde{X}$ in $\tilde{M}$ is tangent to $M$, then the restriction $X$ of $\tilde{X}$ to $M$ is a pseudo-conformal vector field in $M$.

Let $(f, \xi, \theta)$ be an almost contact structure induced in a coordinate neighborhood $U$ of $M$ and assume that $\xi$ is a pseudo-conformal vector field in $U$. Then $(f, \xi, \theta)$ is said to be regular [5]. If this is the case, (5.1) implies

$$
P_{b}{ }^{a}=-\mathcal{L}_{\hat{\xi}} f_{b}{ }^{a}=0,
$$

because $\mathcal{L}_{\xi} \xi=0$ and (5.1) gives $V=0$ and $a=0$. Therefore (3.27) implies

$$
H_{e}{ }^{a} f_{b}{ }^{e}-f_{e}{ }^{a} H_{b}{ }^{e}-l_{b} \xi^{a}=0 \quad\left(\bmod \theta_{b}\right)
$$

if and only if $(f, \xi, \theta)$ is regular. Thus we have, form Theorem 3.3,

THEOREM 5.4. In a real hypersurface $M$ immersed in a complex manifold, an induced almost contact structure of $M$ is normal if and only if it is regular. in [3].

Theorem 5.3 is however a consequence of Theorem 3.1 and a theorem proved

Let $\left(z^{\lambda}\right)$ be a system of complex coordinates in a coordinate neighborhood $\tilde{U}$ 
of the ambient complex manifold $\tilde{M}$. Then we have

LEMMA 5.5. The condition (5.2) is equivalent to each of the following conditions (5.11) and (5.12):

$$
\begin{gathered}
\frac{\partial \tilde{X}^{\lambda}}{\partial \bar{z}^{\mu}}=0 ; \\
d \tilde{X}^{\lambda} \wedge d z^{1} \wedge \cdots \wedge d z^{n+1}=0 .
\end{gathered}
$$

It is easily verified that condition (5.8) is equivalent to (5.7) which is equivalent to (5.5) and hence to (5.4). Thus we have

Lemma 5.6. Condition (5.8) is equivalent to (5.4) or to

$$
d\left(\tilde{X}^{\lambda} \circ i\right) \wedge d\left(z^{1} \circ i\right) \wedge \cdots \wedge d\left(z^{n+1} \circ i\right)=0,
$$

where $\imath: M \rightarrow \tilde{M}$ is the immersion of $M$.

Let there be given a vector field $X$ in $M$ and put $\hat{X}^{h}=X^{a} B_{a}{ }^{h}$, where $X^{a}$ are components of $X$ in $U$. Then we have

LEMMA 5.7. The condition (5.8) for a vector field $X=X^{a} \partial / \partial y^{a}$ tangent to $M$ is equivalent to the condition

$$
d \hat{X}^{\lambda} \wedge d\left(z^{1} \circ i\right) \wedge \cdots \wedge d\left(z^{n+1} \circ i\right)=0,
$$

where $\imath: M \rightarrow \tilde{M}$ is the immersion of $M$ and $\hat{X}^{h}=X^{a} B_{a}{ }^{h}$.

We now assume that $M$ is a real hypersurface analytically immersed in $\tilde{M}$ and that $X$ is an analytic vector field in $M$. Then, as is well known, the differential equation (5.12) with unknown functions $\tilde{X}^{\lambda}\left(z^{\mu}, \bar{z}^{\mu}\right)$ has a local solution $\tilde{X}^{\lambda}$ satisfying the boundary condition

$$
\tilde{X}^{\lambda} \circ i=\hat{X}^{\lambda}
$$

along $M$, when $\hat{X}^{\lambda}$ satisfy condition (5.13) [5]. Therefore, taking account of Theorem 5.3 and Lemmas 5.5, 5.6 and 5.7, we can prove the following theorem[5]:

THEOREM 5.8. Let $M$ be a real hypersurface analytically immersed in a complex manıfold $\tilde{M}$. Then an analytuc vector field $X$ in $M$ is pseudo-conformal if and only if, for any point $P$ belonging to $M$, there are a neighborhood $\tilde{O}$ of $M$ contammg $P$ and a holomorphic vector field $\tilde{X}$ in $\tilde{O}$ such that $X$ is the restriction of $\tilde{X}$ to $\tilde{O} \cap M$.

\section{BIBLIOGRAPHY}

[1] CARTAN, E., Sur la géométrie pseudo-conforme des hypersurfaces des deux variables complexes I, Ann. di Mat. Pura ed Appl. (4) 11 (1932), 17-90 (or Oeuvres, II, 2, 1231-1304) ; II, Ann. Scuola Norm. Pisa, (2) 1 (1932), 333-354 
(or Oeuveres III, 2, 1217-1238).

[2] Chern, S.S. And J.K. Moser, Real hypersurfaces in complex manifolds, Acta Math., 133 (1974), 219-271.

[3] Ishihara, S., Distributions with complex structure, to appear in Kodai Math. J.

[4] SASAKI, S., Almost contact manıfolds, Part I, Lecture Notes, Tôhoku Unıversity, Sendai, Japan, 1965.

[5] TANAKA, N., On the pseudo-conformal geometry of hypersurfaces of the space of complex variables, J. Math. Soc. of Japan, 14 (1962), 397-429.

[6] Walker, A.G., Connexions for parallel distributions in the large, I, Quart. J. of Math., Oxford (2), 6 (1955), 301-308, II, 9 (1958), 221-231.

[7] YANo, K., Differential geometry on complex and almost complex spaces, Pergamon Press, 1965.

Tokyo Institute of Technology 